\title{
The relationship between platelet count and the onset of leaking phase in dengue patients
}

\author{
Nishshanka $\mathbf{P}^{1}$, Kodikararachchi RW ${ }^{2}$, Karunanayake ${ }^{3}$ \\ ${ }^{1}$ Postgraduate Institute of Medicine, Colombo, Sri Lanka. \\ ${ }^{2}$ Department of Clinical Medicine, Teaching Hospital Karapitiya, Galle, Sri Lanka. \\ ${ }^{3}$ Department of Physiology, Faculty of Medicine, University of Ruhuna, Galle, Sri Lanka. \\ Correspondence: Dr.Amaranath Karunanayake \\ e-mail: a2222nath@gmail.com \\ D https://orcid.org/0000-0001-5836-6329 \\ Submitted on 31.05.2021 and accepted for publication on 25.06.2021
}

\begin{abstract}
Introduction: The complications of dengue are related to reduced intravascular volume following plasma leakage. Thrombocytopenia or platelet dysfunctions are detected in all stages of the illness and are related to the clinical outcome. The main objective of the study was to correlate absolute platelet count to the onset of leaking phase in dengue patients who had entered into leaking phase after the hospital admission.
\end{abstract}

Methods: One hundred and sixteen patients (age range 14-74 years, median age 33 years) who entered the leaking phase after admission were recruited to the study. Dengue infection in patients was confirmed with either the presence of positive NS1 or positive IgM antibodies. Serial ultrasonography was done from admission until the leaking phase is detected. Platelet counts were assessed twice per day just prior to the ultrasonography.

Results: There were 47 (40.5\%) females and 69 (59.5\%) males among the recruited patients. The mean number of days at which leaking was detected following onset of fever was $4.9 \pm 0.9$ days. The mean platelet count at the onset of leaking phase was $30,051 \pm 17,023 / \mu \mathrm{L}$. Age and the platelet count at the time of onset of leaking phase was significantly negatively correlated (whole group $\mathrm{r}=-0.323 p<0.001$; in females $\mathrm{r}=-0.369$, $p=0.011$; males $\mathrm{r}=-0.280 p=0.020$ ). Platelet count at the time of onset of leaking phase was significantly lower in the patients older than 30 years of age $(27,577.5 \pm 17,361.4)$ compared to those of the patients younger than 30 years $(33,955.6 \pm 15,888.8)(\mathrm{p}=0.032)$. Upper limit of the range of platelet count at the onset of leaking phase was $77,000 / \mu \mathrm{L}$ which was slightly lower in females.

Conclusions: According to this study, the value of platelet count as a predictor of the progression of dengue infection depends on the age of the patients. Young patients are more prone to develop leaking phase with higher platelet counts than the older patients.

Key words: Thrombocytopaenia, dengue, leaking phase

\section{Introduction}

Dengue viral fever is the most prevalent mosquitoborne disease in the world.

The first case of dengue in Sri Lanka was reported in 1960 (1). During the past few decades, the Sri Lankan population has gone through several epidemics and is currently experiencing annual dengue epidemics (2). The incidence and the severity of the disease are significantly increased during recent years resulting in high mortality.

Many dengue patients are asymptomatic and may not progress into the critical phase. The critical phase 
occurs around the time of defervescence associated with an increased tendency for capillary leakage. The leaking phase manifests as a rise in haematocrit, pleural effusions, ascites or haemorrhage (3).

Therefore, dengue patients should be closely monitored for the development of plasma leakage. As a prognostic indicator, ultra sound scans (USS) could be used to assess patients at risk for entering the critical phase. Gallbladder wall thickening due to oedema which can be detected in USS is considered the earliest sign of plasma leaking in dengue haemorrhagic fever (4). The haematological parameters are useful in predicting the development of critical phase within the next 24 to 48 hours (5).

The platelet count has value as potential indicator of clinical severity in dengue $(6,7)$. A rapid decline in platelet count or a platelet count less than $150,000 / \mu \mathrm{L}$ blood is an alarming sign of dengue (6). The mechanisms involved in thrombocytopenia are not fully understood (8). Bone marrow suppression $(9,10)$ and increased peripheral destruction $(11,12)$ are the proposed mechanisms for thrombocytopenia. Furthermore, number of studies have documented platelet dysfunction in dengue $(13,14)$.

\& The value of platelet count as a predictor of critical phase has been evaluated in several studies. The rapid decline of platelet counts before the critical phase has been reported in both adult and paediatric studies (15-17). Several studies showed gallbladder wall thickening which was significantly associated with lower platelet counts (17-20). Santhosh et al. have reported gallbladder wall oedema in $97.8 \%$ of patients whose platelet count was less than $40,000 / \mu \mathrm{L}(19)$.

There have been no studies done in Sri Lanka on the value of platelet count as a predictor of the critical phase. The main objective of this study was to assess the relationship between ultrasonographic evidence of earliest leakage and corresponding platelet count in dengue patients who had entered into leaking phase after the hospital admission.

\section{Methods}

This descriptive cross-sectional study was conducted at Teaching Hospital, Karapitiya, over a period of six months. All patients with confirmed dengue fever with positive NS1 antigen or IgM antibodies and developed leaking phase after the admission into two medical wards were included in the study. Patients with dengue leaking phase on admission, patients with other aetiologies for thrombocytopenia such as cirrhosis, splenomegaly, autoimmune disorders, myelodysplastic syndromes or malignancies and patients who were on thrombocytopenic drugs were excluded from the study.

After obtaining informed written consent, the data were collected by the principal investigator using a pre-tested questionnaire.

All dengue patients underwent serial ultrasonography on admission and then twice a day until leaking phase was over. Ultrasonography was performed by a medical registrar experienced in detecting dengue leaking phase under the supervision of a consultant radiologist. The consultant radiologist repeated all doubtful scans. USS studies were performed with 6 hours of fasting to optimize gallbladder visualization. Gallbladder wall thickening was used as a radiological sign to detect the leaking phase. Wall thickness of more than $3 \mathrm{~mm}$ was considered as gallbladder wall thickening (18). All ultrasound examinations were performed with a portable machine ( $L \& T$ symphony model) using $3.5 \mathrm{MHz}$ and $5 \mathrm{MHz}$ probes. Gallbladder wall thickness was measured by using a previously reported and validated ultrasound method (21). Blood samples were collected 30 minutes before each scan using standard procedures in to EDTA tubes and immediately analysed using Mindry 6068 and Sysmex XN 1000 automated full blood count analysers. Once the leaking was detected latest platelet count was taken for the analysis.

The data were analysed with PSPP version 0.83g5f9212 statistical software (Free Software Foundation, Inc. htttp//fsf.org/). Mann Whitney U test was used to assess the significance between variables. $P<0.05$ was considered statistically significant.

The ethical approval for the study was obtained from the Ethics Review Committee of Faculty of Medicine, University of Ruhuna. Approval was taken from the Director, Teaching Hospital, Karapitiya for data collection and written, informed consent was obtained from the patients or the guardian in case of patients less than 18 years of age. 


\section{Results}

Of one hundred sixteen patients who had entered into leaking phase after the hospital admission, 47 $(40.5 \%)$ were females and $69(59.5 \%)$ were males. The median age of females was 36 years and males were 32 years. Majority (55\%) of the patients were from Galle Municipality area. Demographic characteristics and variables related to the disease course are given in Table 1.

The duration of hospital stay was significantly higher in female patients than in male patients $(p=0.043)$. However, gender and the duration of the fever on admission was negatively correlated with the duration of the hospital stay $(\mathrm{r}=-0.344$; $p=0.018)$. Furthermore, there was no gender-related difference in the duration of fever on admission.

The duration of fever on admission was negatively correlated with the duration of hospital stay $(\mathrm{r}=$ $-0.410 ; p<0.001)$ and platelet count at the onset of leaking phases $(\mathrm{r}=-0.200 ; p=0.031)$. Furthermore, the duration of fever on admission was positively correlated with the duration of fever at the onset of leaking phase $(\mathrm{r}=0.201 ; p=0.031)$.

However, among the male patients, the duration of fever on admission was negatively correlated with the mean duration of hospital stay $(r=-0.490$; $p<0.001)$ and mean platelet count at the onset of leaking phases $(\mathrm{r}=-.331 ; p=0.005)$. The platelet count at the onset of leaking phases was positively correlated with the duration of the hospital stay $(\mathrm{r}=-0.252 ; p=0.037)$.

Correlation of the mean duration of fever on admission, during the hospital stay and at the onset of leaking phase according to the age group is given in Figure 1.

The mean number of days the leaking phase was detected after the onset of fever was $4.9 \pm 0.9$ days. There was no statistically significant association between the duration of fever at the onset of leaking phase and gender ( $p=0.247)$ (Figure 2). Mean platelet count at the onset of leaking phase was $30,051 \pm 17,023$. There was no significant association of platelet count at the onset of leaking phase and the gender $(p=0.478)$.

There was a significant negative correlation between the age and the platelet count at the time of onset of leaking phase in the sample $(\mathrm{r}=-0.323 p=<0.001$; females $\mathrm{r}=-0.369 ; p=0.011$ and males $\mathrm{r}=-0.280$; $p=0.020$ ) (Figure 3). Upper limit of the range of platelet count at the onset of leaking phase was slightly lower in females.

Table 1: Demographic characteristics and variables related to the disease course

\begin{tabular}{|c|c|c|c|}
\hline Demographic characteristics & $\begin{array}{l}\text { Male } \\
(n=69)\end{array}$ & $\begin{array}{l}\text { Female } \\
(n=47)\end{array}$ & $\begin{array}{l}\text { Total } \\
(n=116)\end{array}$ \\
\hline Age (median)in years & 32 & 36 & 33 \\
\hline Age range (years) & $14-69$ & $15-74$ & $14-74$ \\
\hline Mean duration (SD) of the hospital stay (days) & $5.1(1.4)$ & $5.6(1.5)$ & $5.3(1.5)$ \\
\hline Range of duration of hospital stay (days) & $3-10$ & $4-10$ & $3-10$ \\
\hline $\begin{array}{l}\text { Mean duration (SD) of fever in days on } \\
\text { admission }\end{array}$ & $4.0(1.5)$ & $4.2(1.6)$ & $4.1(1.5)$ \\
\hline $\begin{array}{l}\text { Mean duration (SD) of fever in days at the } \\
\text { onset of leaking phase }\end{array}$ & $4.8(0.9)$ & $5.0(0.9)$ & $4.9(0.9)$ \\
\hline $\begin{array}{l}\text { Mean platelet count (cells/ } \mu \mathrm{L} \text { ) (SD) at the } \\
\text { onset of leaking phase }\end{array}$ & $30449(16554)$ & $29468(17855)$ & $30051(17023)$ \\
\hline $\begin{array}{l}\text { Range of platelet count at the onset of leaking } \\
\text { phase (cells } / \mu \mathrm{L})\end{array}$ & $3,000-77,000$ & $7,000-74,000$ & $3,000-77,000$ \\
\hline
\end{tabular}




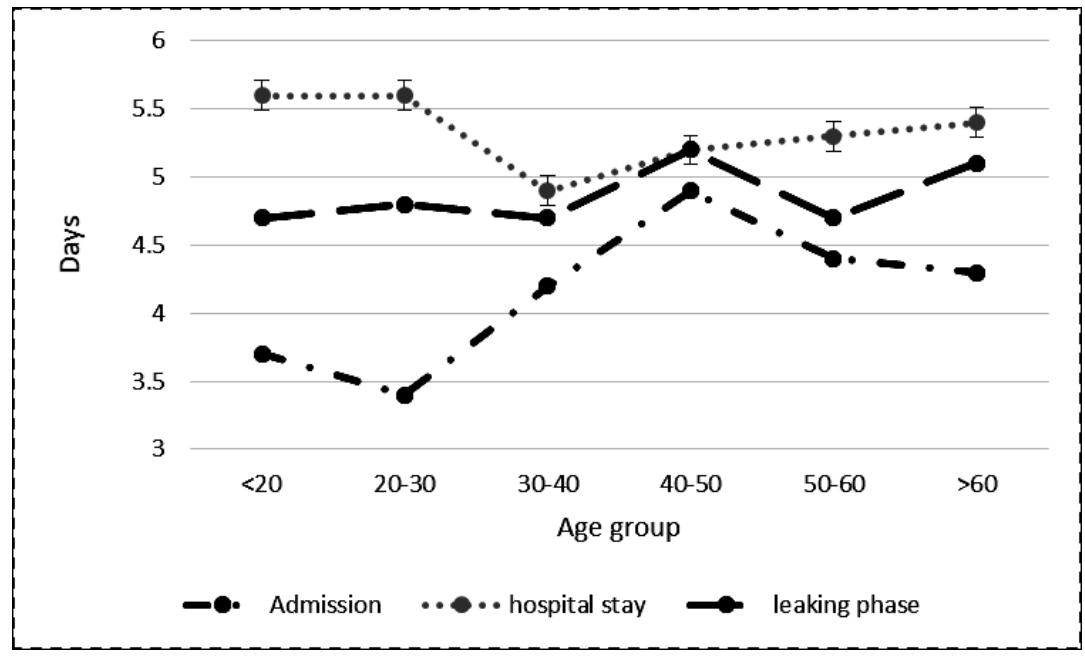

Figure 1: Mean durations of fever; on admission, during hospital stay and at the onset of leaking phase according to the age group

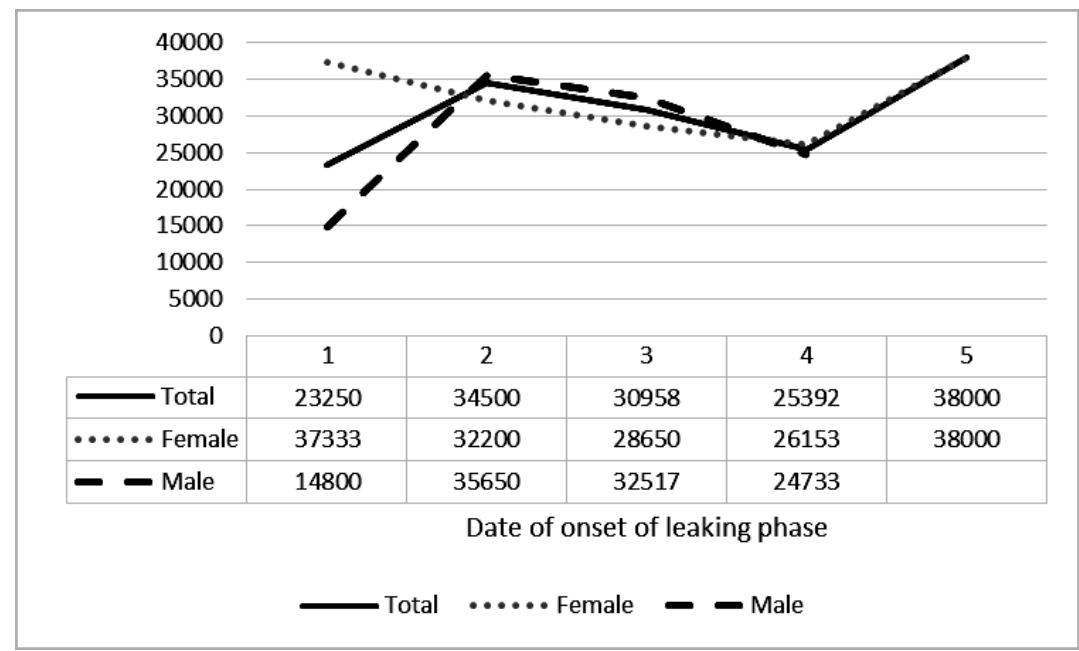

Figure 2: Correlation between mean platelet count at the onset of leaking phase and mean duration of the fever at the onset of leaking phase

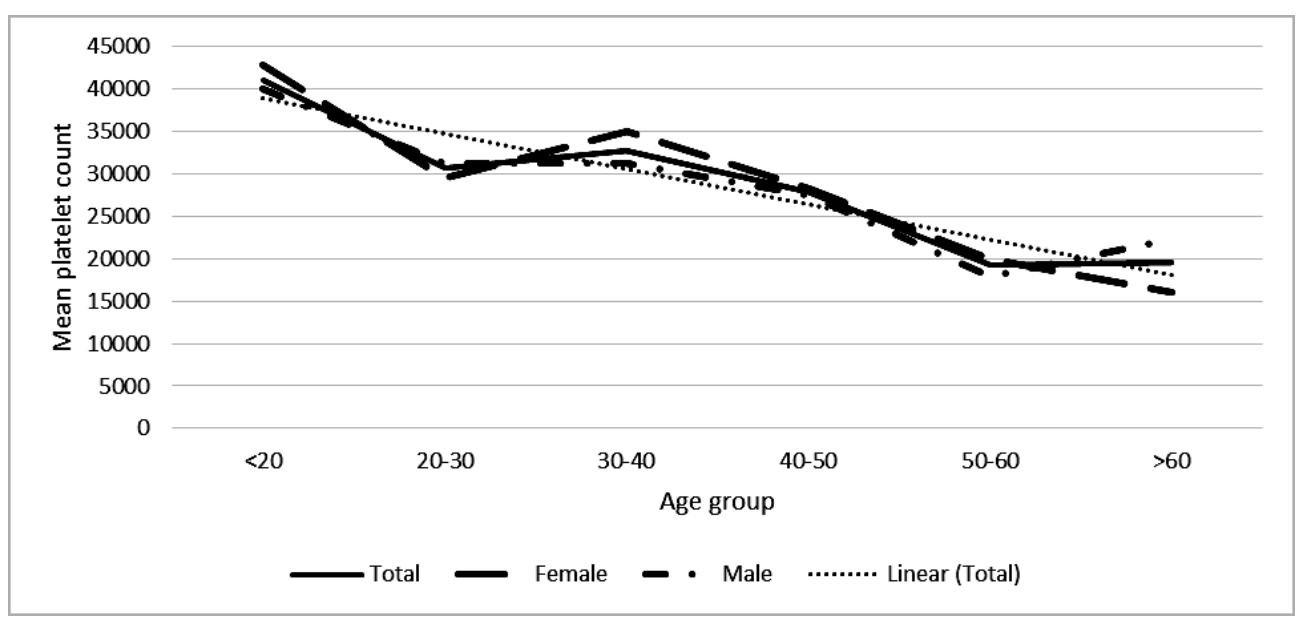

Figure 3: Correlation between age and the platelet count at the time of onset of leaking phase 
The platelet count at the time of onset of leaking phase was significantly lower in patient older than 30 years of age $(27,577.5$, SD 17,361.4) than patients younger than 30 years of age $(33,955.6$, SD 15, 888.8 $)(p=0.032)$.

\section{Discussion}

This study shows that the value of platelet count as a predictor of the progression of dengue fever depends on the age of the patients.

Age and gender have been studied as factors forecasting the severity of dengue. Female gender and young age have been found as risk factors for mortality (22). The duration of hospital stay was significantly longer in female patients than male patients in the current study which would support the finding of preponderance of females among severe dengue cases but not among mild cases (23). It has been suggested that risk of female gender reflects either a more robust cellular immune response or a higher intrinsic susceptibility to capillary permeability in females than males (24).

The age-stratified analyses in previous studies have demonstrated the difference in clinical and laboratory manifestations of dengue between elderly and younger adults. It was observed that children less than 10 years of age are more prone to increased vascular permeability, leading to shock (24). In our cohort, the age and the platelet count at the time of onset of leaking phase was significantly negatively correlated. This shows that the young patients are more prone to develop leaking phase with higher platelet counts than the older patients.

Similar to our findings, several studies have reported that platelet count $\leq 100,000 / \mu \mathrm{L}$ increased the risk of leaking $(25,26)$. Furthermore, platelet count decreases rapidly before patients enter the state of shock. This emphasizes that in young patients leaking phase may develop at higher platelet counts than in elderly patients.

Upper limit of the range of platelet count at the onset of leaking phase was in this study was $77,000 / \mu \mathrm{L}$, which was slightly lower in females. Considering the higher limit of the mean platelet count at the onset of leaking phase as observed in the current study, we propose that the leaking phase must be anticipated when the platelet count reaches $80,000 / \mu \mathrm{L}$. National Guidelines recommend that platelet counts less than $100,000 / \mu \mathrm{L}$ indicate that the patient might enter the critical phase (onset of plasma leakage) and this is supported by many other studies $(27,29)$. This study further supports the above recommendation.

The mean duration of fever at the onset of the leaking phase was $4.9 \pm 0.9$ days with a wide range in platelet count. These findings emphasize the importance of early detection and continuous monitoring of dengue patients. Early identification of the patients with risk improves the outcome. Furthermore, it may enhance the efficient allocation of hospital resources by diverting resources for patient with impending threat for severe disease.

\section{Conclusions}

The value of platelet count as a predictor of the progression of dengue fever depends on the age of the patients. Younger patients are more prone to develop leaking phase with higher platelet counts than the older patients.

\section{References}

1. Munasinghe DR, Amarasekera PJ, Fernando CF. An epidemic of dengue-like fever in Ceylon (chikungunya a clinical and haematological study. Ceylon Med J. 1966; 11(4): 129-142.

2. Sirisena PDNN, Noordeen F. Evolution of dengue in Sri Lanka-changes in the virus, vector, and climate. International Journal of Infectious Diseases. 2014; 19: 6-12.

3. Srikiatkhachorn A. Plasma leakage in dengue haemorrhagic fever. Thromb Haemost. 2009; 102(6): 1042 1049.

4. Colbert JA, Gordon A, Roxelin R, Silva S, Silva J, Rocha C, et al. Ultrasound measurement of gallbladder wall thickening as a diagnostic test and prognostic indicator for severe dengue in pediatric patients. The Pediatric Infectious Disease Journal. 2007; 26(9): 850-852.

5. Ministry of Health Sri Lanka icwCCoP. Guidelines on clinical management of dengue fever and dengue haemorrhagic fever in adults. Sri Lanka. Ministry of Health Sri Lanka, 2010. 
6. World Health O. Dengue guidelines for diagnosis, treatment, prevention and control: new edition. Geneva: World Health Organization, 2009.

7. Jayashree K, Manasa GC, Pallavi P, Manjunath GV. Evaluation of platelets as predictive parameters in dengue Fever. Indian J Hematol Blood Transfus. 2011; 27(3): 127-130.

8. de Azeredo EL, Monteiro RQ, de-Oliveira Pinto LM. Thrombocytopenia in Dengue: Interrelationship between Virus and the Imbalance between Coagulation and Fibrinolysis and Inflammatory Mediators. Mediators Inflamm. 2015;2015:313842.

9. Murgue B, Cassar O, Guigon M, Chungue E. Dengue virus inhibits human hematopoietic progenitor growth in vitro. The Journal of Infectious Diseases. 1997; 175(6): $1497-$ 501.

10. Basu A, Jain P, Gangodkar SV, Shetty S, Ghosh K. Dengue 2 virus inhibits in vitro megakaryocytic colony formation and induces apoptosis in thrombopoietininducible megakaryocytic differentiation from cord blood CD34 + cells. FEMS Immunology and Medical Microbiology. 2008; 53(1): 46-51.

11. Srichaikul T, Nimmannitya S. Haematology in dengue and dengue haemorrhagic fever. Baillieres Best Pract Res Clin Haematol. 2000; 13(2): 261-276.

12. Mitrakul C. Bleeding problem in dengue haemorrhagic fever: platelets and coagulation changes. The Southeast Asian Journal of Tropical Medicine and Public Health. 1987; 18(3): 407-412.

13. Matsuura C, Moraes TL, Barbosa JB, Moss MB, Siqueira MA, Mann GE, et al. Nitric oxide activity in platelets of dengue haemorrhagic fever patients: the apparent paradoxical role of ADMA and 1-NMMA. Transactions of the Royal Society of Tropical Medicine and Hygiene. 2012; 106(3): 174-179.

14. Michels M, Alisjahbana B, De Groot PG, Indrati AR, Fijnheer R, Puspita M, et al. Platelet function alterations in dengue are associated with plasma leakage. Thrombosis and Haemostasis. 2014; 112(2): 352-362.

15. Ralapanawa U, Alawattegama ATM, Gunrathne M, Tennakoon S, Kularatne SAM, Jayalath T. Value of peripheral blood count for dengue severity prediction. BMC Res Notes. 2018; 11(1): 400
16. Lam PK, Ngoc TV, Thu Thuy TT, Hong Van NT, Nhu Thuy TT, Hoai Tam DT, et al. The value of daily platelet counts for predicting dengue shock syndrome: Results from a prospective observational study of 2301. Vietnamese children with dengue. PLoS Neglected Tropical Diseases. 2017; 11(4): e0005498.

17. Sudhir Sachar SG, Saurabh Sacha. Role of Ultrasonography ("Honeycomb Sign") in Early Detection of Dengue Hemorrhagic Fever. Arch Clinexp Surg. 2013; 2(1): 38-42.

18. Venkata Sai PM, Dev B, Krishnan R. Role of ultrasound in dengue fever. Br J Radiol. 2005; 78(929): 416-418.

19. Santhosh VR, Patil PG, Srinath MG, Kumar A, Jain A, Archana M. Sonography in the diagnosis and assessment of dengue Fever. Journal of Clinical Imaging Science. 2014; $4: 14$

20. Kanaga DS, Natarajan S, Nanjundan M, Balamurugan PP. Role of Ultrasonography in Diagnosing and Predicting the Severity of Dengue Fever. IOSR Journal of Dental and Medical Sciences (IOSR-JDMS). 2016; 15(12): 43-50.

21. Shlaer WJ, Leopold GR, Scheible FW. Sonography of the thickened gallbladder wall: a nonspecific finding. American Journal of Roentgenology. 1981; 136(2): $337-$ 339.

22. Anders KL, Nguyet NM, Chau NV, Hung NT, Thuy TT, Lien le B, et al. Epidemiological factors associated with dengue shock syndrome and mortality in hospitalized dengue patients in Ho Chi Minh City, Vietnam. The American Journal of Tropical Medicine and Hygiene. 2011; 84(1): 127-134.

23. Halstead SB, Nimmannitya S, Cohen SN. Observations related to pathogenesis of dengue hemorrhagic fever. IV. Relation of disease severity to antibody response and virus recovered. The Yale Journal of Biology and Medicine. 1970; 42(5): 311-328.

24. Halstead SB. Epidemiology of dengue and dengue hemorrhagic fever Gubler DJ KG, editor. Oxon, UK: CABI, 1997.

25. Pham TB, Nguyen TH, Vu TQ, Nguyen TL, Malvy D. Predictive factors of dengue shock syndrome at the children hospital No. 1, Ho-chi-Minh City, Vietnam. Bulletin de la Societe de Pathologie Exotique. (1990). 2007; 100(1): 43-47. 
26. Chuansumrit A, Puripokai C, Butthep P, Wongtiraporn W, Sasanakul W, Tangnararatchakit K, et al. Laboratory predictors of dengue shock syndrome during the febrile stage. The Southeast Asian Journal of Tropical Medicine and Public Health. 2010; 41(2): 326-332.

27. Potts JA, Rothman AL. Clinical and laboratory features that distinguish dengue from other febrile illnesses in endemic populations. 2008; 13(11): 1328-40.
28. Krishnamurti C, Kalayanarooj S, Cutting MA, Peat RA, Rothwell SW, Reid TJ, et al. Mechanisms of hemorrhage in dengue without circulatory collapse. $J$ The American Journal of Tropical Medicine and Hygiene. Am J Trop Med Hyg Am. J. Trop. Med. Hyg. 2001; 65(6): 840-847.

29. Wills B, Van Ngoc T, Van NTH, Thuy TTT, Thuy TTN, Dung NM, et al. Hemostatic Changes in Vietnamese Children with Mild Dengue Correlate with the Severity of Vascular Leakage Rather than Bleeding. The American Journal of Tropical Medicine and Hygiene Am J Trop Med Hyg. 2009; 81(4): 638-644. 